\title{
近球状钼酸镍/多壁碳纳米管复合材料的制备及其噟电容性能
}

\author{
刘甜甜，王庆华，刘希莉，高 凤，汪庆祥
}

(闽南师范大学化学化工与环境学院, 漳州 363000)

摘 要: 以硝酸镍、钼酸钠和多壁碳纳米管为原料, 通过水热反应法制备了钼酸镍/多壁碳纳米管 $\left(\mathrm{NiMoO}_{4} / \mathrm{MWCNTs}\right)$ 复合材料。采用扫描电子显微镜、X 射线能谱和 X 射线衍射对材料组成和形貌进行表征。结果表明: MWCNTs 很 好地包覆在球状 $\mathrm{NiMoO}_{4}$ 外表面, 且各元素均匀地分布在材料中。循环伏安和电化学阻抗实验证实 MWCNTs 显著 增强了 $\mathrm{NiMoO}_{4}$ 的氧化还原信号和电荷转移动力学特性。电容测试实验进一步表明, 复合材料较 $\mathrm{NiMoO}_{4}$ 单一材料 具有更高的比电容、倍率特性及循环稳定性, 且当 MWCNT 含量为 $40 \mathrm{mg}$ 时, 所得产物 $\left(\mathrm{NiMoO}_{4} / \mathrm{MWCNTs}-40\right)$ 具有 最佳的电化学性能。电流密度为 $2 \mathrm{~A} / \mathrm{g}$ 时, $\mathrm{NiMoO}_{4} / \mathrm{MWCNTs}-40$ 复合材料的比电容高达 $1071 \mathrm{~F} / \mathrm{g}$; 当电流密度增大 到 $10 \mathrm{~A} / \mathrm{g}$ 时, 比电容仍能保持原来的 $66.10 \%$ 。在 $10 \mathrm{~A} / \mathrm{g}$ 的电流密度下, 经过 2500 次循环充放电后, $\mathrm{NiMoO}_{4} /$ MWCNTs-40 复合材料的比电容保持率高达 $95.85 \%$, 表明该材料具有出色的循环稳定性。

关 键 词: 钼酸镍; 多壁碳纳米管; 复合材料; 超级电容器

中图分类号: TM535 文献标识码: A

\section{Synthesis and Pseudocapacitive Behavior of Nickel Molybdate/Mutiwalled Carbon Nanotubes Composite}

\author{
LIU Tian-Tian, WANG Qing-Hua, LIU Xi-Li, GAO Feng, WANG Qing-Xiang \\ (College of Chemistry and Environment, Minnan Normal University, Zhangzhou 363000, China)
}

\begin{abstract}
A novel nickel molybdate/mutiwalled carbon nanotubes ( $\left.\mathrm{NiMoO}_{4} / \mathrm{MWCNTs}\right)$ composite was synthesized by hydrothermal method using nickel nitrate, sodium molybdate and MWCNTs as raw materials, and the product was characterized by scanning electronic microscopy, energy dispersive spectra, and X-ray diffraction spectra. The scanning electron microscopy results showed that pseudo-spherical $\mathrm{NiMoO}_{4}$ was wrapped with MWCNTs and all elements were uniformly distributed in the material. Cyclic voltammetry and electrochemical impedance spectra revealed that the presence of MWCNTs greatly improved the redox signal and electron transfer kinetics of $\mathrm{NiMoO}_{4}$. The capacitance tests further indicated that the composite presented better specific capacitance, rate capability, and cycling stability than the single-component of $\mathrm{NiMoO}_{4}$, and with a MWCNTs content of $40 \mathrm{mg}$ the composite $\left(\mathrm{NiMoO}_{4} / \mathrm{MWCNTs}^{-40}\right)$ displayed the best electrochemical performance. At the current density of $2 \mathrm{~A} / \mathrm{g}$, the $\mathrm{NiMoO}_{4} / \mathrm{MWCNTs} 40$ composite demonstrated a remarkable specific capacitance of $1071 \mathrm{~F} / \mathrm{g}$; while current density was increased to $10 \mathrm{~A} / \mathrm{g}$ and the capacitance still remained $66.10 \%$. Cyclic stability experiment revealed that the $\mathrm{NiMoO}_{4} / \mathrm{MWCNTs}-40$ composite retained $95.85 \%$ of its initial specific capacitance after 2500 charge-discharge cycles at a current density of $10 \mathrm{~A} / \mathrm{g}$, exhibiting prominent electrochemical stability.
\end{abstract}

收稿日期: 2017-09-12; 收到修改稿日期：2017-12-19

基金项目：国家自然科学基金(21275127); 福建省中青年教师教育科研项目(JA15305)

National Natural Science Foundation of China (21275127); Education-Science Research Project for Young and Middle-aged Teachers of Fujian (JA15305)

作者简介: 刘甜甜(1992-), 女, 硕士研究生. E-mail: liutiantian0825@outlook.com

通讯作者: 汪庆祥, 教授. E-mail: axiang236@126.com 
Key words: nickel molybdate; mutiwalled carbon nanotubes; composite; supercapacitor

在现代工业社会, 由于日益增长的能源需求, 不可再生资源的储备量日益减少, 能源危机成为社 会经济发展越来越严重的障碍。超级电容器作为一 种高效的能量储存和转化器件具有充电时间短、库 仑效率高、循环寿命长和环境友好等特点 ${ }^{[1]}$, 在新能 源开发 ${ }^{[2]}$ 、电子产品 ${ }^{[3]}$ 和电动汽车发展 ${ }^{[4]}$ 等领域显示 出了广阔的应用前景。

电极材料是决定电容器性能的关键性因素 ${ }^{[5]}$, 而比电容和循环稳定性是评价电极材料的重要指 标。过渡金属化合物具有较高的噟电容, 但其循环 寿命短，在多次充放电后，材料的氧化还原反应可 逆性恶化, 电子储存能力下降, 比电容降低 ${ }^{[6]}$ 。导电 聚合物具有较强的柔韧性, 但其导电性差, 且种类 有限, 很难得到高比电容的导电聚合物 ${ }^{[7]}$ 。碳材料是 应用最早、工业化产量最高的电极材料, 它具有比 表面积大、导电性好、来源广泛和成本低的特点 ${ }^{[8-9]}$, 常见的碳材料有活性炭、石墨烯、碳纳米管和碳气 凝胶等。然而单纯的碳材料通过双电层储能, 比电 容较小。因此, 为了得到兼具高稳定性和大比电容 的电极材料, 常将过渡金属化合物与碳材料复合, 从而得到兼具二者优秀性能的复合电极材料。比如, $\mathrm{Liu}$ 等 ${ }^{[10]}$ 曾在 $\mathrm{NiMoO}_{4}$ 纳米棒中引入还原氧化石墨 烯(rGO), 得到二者的复合材料。经过 1000 次循环 后，容量保持率是 $84.6 \%$, 相比于单组分 $\mathrm{NiMoO}_{4}$ $71.5 \%$ 的保持率, 有明显提高。

碳纳米管作为一种经典的一维碳纳米材料, 具 有结晶度高、导电性好和比表面积大等优点 ${ }^{[11]}$ 。当 其与金属氧化物形成复合材料, 不仅可以增强导电 性，还可以提高材料稳定性 ${ }^{[12]}$ 。Zhou 等 ${ }^{[13]}$ 曾在碳纳 米管上生长过渡金属氧化物, 得到 $\mathrm{NiCoO}_{2} @ \mathrm{CNT}$ 复合材料。电化学实验显示其比电容约为 $\mathrm{NiCoO}_{2}$ 的两倍, 在 $10 \mathrm{~A} / \mathrm{g}$ 下经过 3000 次循环, 比电容保持 率为 $98.6 \%$, 表现出优异的电化学性能及循环稳定 性，但其合成方法复杂。本课题组以 MWCNTs、硝 酸镍、钼酸钠和六次甲基四胺为主要原料, 通过简 单的一步水热法合成 MWCNTs 包覆 $\mathrm{NiMoO}_{4}$ 的球形 复合物 $\left(\mathrm{NiMoO}_{4} / \mathrm{MWCNTs}\right)$, 并采用扫描电子显微 镜和 $X$ 射线衍射对产物组成、形貌进行了表征。进 一步以此材料为超级电容器的电极材料, 采用循环 伏安、电化学阻抗和恒电流充放电实验等考察了 $\mathrm{NiMoO}_{4} / \mathrm{MWCNTs}$ 的电化学性能。

\section{1 实验方法}

\section{1 试剂与仪器}

六水合硝酸镍 $\left(\mathrm{Ni}\left(\mathrm{NO}_{3}\right)_{2} \cdot 6 \mathrm{H}_{2} \mathrm{O}\right.$, 西陇化工股份 有限公司); 二水合钼酸钠 $\left(\mathrm{Na}_{2} \mathrm{MoO}_{4} \cdot 2 \mathrm{H}_{2} \mathrm{O}\right.$, 西陇化 工股份有限公司); 六次甲基四胺(上海久亿化学试 剂有限公司); 炭黑(日本碳素株式会社); 聚四氟乙 烯浓缩分散液(上海阿拉丁生化科技股份有限公司); 泡沫镍(长沙力元新材料股份有限公司); 多壁碳纳 米管(MWCNTs，深圳市纳米港有限公司)。

JEOL JSM-6010LA 扫描电子显微镜(日本电子 株式会社); D8 Advance- $X$ 射线衍射仪(德国 Bruker 公司); TG209F1 热重分析仪(德国耐驰仪器制造有 限公司); CHI 660E 电化学工作站(上海辰华仪器公 司); CT2001A 电池测试系统(武汉市蓝电电子股份 有限公司); KQ5200E 超声波清洗器(中国上海超声 仪器有限公司)。

\section{2 材料的制备}

$\mathrm{NiMoO}_{4} / \mathrm{MWCNTs}$ 复合材料的制备方法如下: 首先, 将 $0.8 \mathrm{~g} \mathrm{MWCNTs}$ 加入 $50 \mathrm{~mL} \mathrm{HNO}$ 和 $\mathrm{H}_{2} \mathrm{SO}_{4}$ 的混合溶液中(体积比 $2: 3$ ), $140^{\circ} \mathrm{C}$ 回流 $2 \mathrm{~h}$ 对碳纳 米管进行羧基化处理; 然后将羧化后的碳纳米管洗 涤至中性, 干燥备用 ${ }^{[14-15]}$ 。将 20、40、60 mg 羧化后 的多壁碳纳米管分别置于 $60 \mathrm{~mL} \mathrm{H}_{2} \mathrm{O}$ 中, 超声 $0.5 \mathrm{~h}$ 至完全分散。然后称取 $1 \mathrm{mmol} \mathrm{Ni}\left(\mathrm{NO}_{3}\right)_{2} \cdot 6 \mathrm{H}_{2} \mathrm{O}, 2 \mathrm{mmol}$ $\mathrm{Na}_{2} \mathrm{MoO}_{4} \cdot 2 \mathrm{H}_{2} \mathrm{O}, 6 \mathrm{mmol}$ 六次甲基四胺, 依次加入多 壁碳纳米管分散液中, 磁力搅拌使其溶解完全。再 将混合液转移至 $100 \mathrm{~mL}$ 内祄聚四氟乙烯的不锈钢 高压釜中密封, $100^{\circ} \mathrm{C}$ 烘箱中反应 $12 \mathrm{~h}$, 自然冷却至 室温, 用乙醇和蒸馏水离心, 洗涤掉未反应的原料, 在 $60^{\circ} \mathrm{C}$ 下真空干燥, 得到 $\mathrm{NiMoO}_{4} / \mathrm{MWCNTs}$ 复合材 料, 分别标记为 $\mathrm{NiMoO}_{4} / \mathrm{MWCNTs}-20, \mathrm{NiMoO}_{4} /$ MWCNTs-40, $\mathrm{NiMoO}_{4} / \mathrm{MWCNTs-60。}$

为进行对比试验, 除了不加碳纳米管之外, 在 其他反应条件都相同情况下, 制得纯 $\mathrm{NiMoO}_{4}$ 材料。

\section{3 电化学测试}

电化学性能测试采用传统的三电极体系: 以铂 丝为对电极, 饱和甘录电极 (SCE) 为参比电极, 将活 性物质涂覆在泡沫镍上作为工作电极。泡沫镍分别 用丙酮、水和乙醇超声, 在真空干燥箱内干燥、待 用。电极活性材料、碳黑与聚四氟乙烯乳液按 
$8: 1: 1$ 的质量比, 以乙醇为分散剂, 在玛瑙研钵中 混合研磨至浆状后, 均匀地涂覆在泡沫镍上, 真空 干燥后压片, 得到 $1 \mathrm{~cm} \times 1 \mathrm{~cm}$ 的待测电极, 活性物 质的负载量约为 $3 \mathrm{mg}$ 。采用 CHI660E 电化学工作 站进行交流阻抗测试(EIS)、循环伏安测试(CV)和恒 电流充放电测试(GCD)。采用 CT2001A 电池测试系 统进行循环稳定性测试。EIS 测试频率范围为 $0.01 \mathrm{~Hz} 100 \mathrm{kHz}$, 振幅为 $5 \mathrm{mV}$ 。 $\mathrm{CV}$ 测试在 $-0.1 \sim$ $0.6 \mathrm{~V}$ 范围内于不同的扫描速率下进行; GCD 测试 在 $0 \sim 0.45 \mathrm{~V}$ 范围内于不同电流密度下进行。所有测 试均以 $2 \mathrm{~mol} / \mathrm{L} \mathrm{KOH}$ 溶液为电解液。比电容通过以 下公式进行计算:

$$
C_{\mathrm{m}}=\frac{C}{m}=\frac{I \times t}{\Delta V \times m}
$$

其中 $C_{\mathrm{m}}(\mathrm{F} / \mathrm{g})$ 是比容量; $I(\mathrm{~A})$ 是放电电流; $m(\mathrm{~g})$ 是 $\mathrm{NiMoO}_{4} / \mathrm{MWCNTs}$ 复合材料的质量; $t(\mathrm{~s})$ 是电容器的 放电时间; $\Delta V(\mathrm{~V})$ 为放电过程电位窗口。

\section{2 结果与讨论}

\section{1 材料表征}

图 1 为 $\mathrm{NiMoO}_{4}(\mathrm{a}) \sim(\mathrm{b})$ 和 $\mathrm{NiMoO}_{4} / \mathrm{MWCNTs}-40$ (c) (d) 在不同分辨率下的 SEM 照片。由图可知, 纯 $\mathrm{NiMoO}_{4}$ 呈近球状结构, 粒径为 $1 \sim 3 \mu \mathrm{m}$ (图 1(a))。高 分辨 SEM 照片表明, 近球状 $\mathrm{NiMoO}_{4}$ 表面具有明显 的褶皱结构。与纯 $\mathrm{NiMoO}_{4}$ 相比, $\mathrm{NiMoO}_{4} / \mathrm{MWCNTs}$ 40 粒径增大至 1.4 3.2 $\mu \mathrm{m}$ 。高分辨 SEM 照片进一 步显示，与纯 $\mathrm{NiMoO}_{4}$ 表面结构不同, $\mathrm{NiMoO}_{4} /$ MWCNTs-40 复合物的表面被一维线状材料包覆, 表明在合成复合材料的过程中, MWCNTs 包覆在了 $\mathrm{NiMoO}_{4}$ 外围。本课题组预测在该结构中, MWCNTs
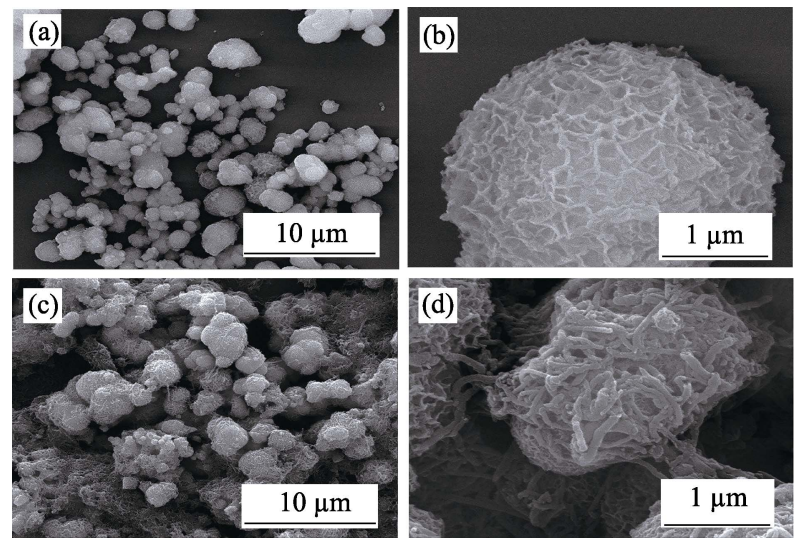

图 1 (a) (b) $\mathrm{NiMoO}_{4}$ 和(c) (d) $\mathrm{NiMoO}_{4} / \mathrm{MWCNTs}-40$ 在不同 分辨率下的扫描电镜照片

Fig. 1 SEM images of (a, b) $\mathrm{NiMoO}_{4}$ and (c, d) Ni$\mathrm{MoO}_{4} / \mathrm{MWCNTs}-40$ at different resolution
一方面能提高内核 $\mathrm{NiMoO}_{4}$ 的电子转移能力, 另一 方面能对 $\mathrm{NiMoO}_{4}$ 化学稳定性起到增强作用。

为了进一步表征复合材料的化学组成及元素分 布，采用 EDS 面扫描对材料进行了表征。图 2(b) (e) 为图 2(a)微面下对应的 $\mathrm{NiMoO}_{4} / \mathrm{MWCNTs}-40$ EDS 能谱图, 从图中可以看出, $\mathrm{Ni} 、 \mathrm{Mo} 、 \mathrm{O} 、 \mathrm{C}$ 四种元素 都均匀分布于样品中。图 2(f)为不同 MWCNTs 含量 $(0 \sim 60 \mathrm{mg})$ 的 $\mathrm{NiMoO}_{4} / \mathrm{MWCNTs}$ 复合材料的 XRD 图 谱。由图可见, 在 $36^{\circ}$ 和 $61^{\circ}$ 附近出现的两个衍射峰, 属于 $\mathrm{NiMoO}_{4}$ 的特征衍射峰 ${ }^{[10]}$ 。该衍射峰很宽且弱, 说明 $\mathrm{NiMoO}_{4}$ 结晶度较低。加入碳管以后, 在 $26^{\circ}$ 附近出现一个宽峰, 属于碳管的(002)衍射峰。随着 碳管含量的增加, 碳峰也逐渐增强, 其他峰基本保 持不变, 说明在碳管与 $\mathrm{NiMoO}_{4}$ 结合的过程中, 并 未对 $\mathrm{NiMoO}_{4}$ 的晶型产生影响。

\section{2 电化学阻抗}

图 3 是 $\mathrm{NiMoO}_{4}$ 和 $\mathrm{NiMoO}_{4} / \mathrm{MWCNTs}-40$ 复合材 料的交流阻抗图谱。由图可见，在高频区 (插图) $\mathrm{NiMoO}_{4} / \mathrm{MWCNTs}-40$ 复合材料与实轴交点的截距 小于 $\mathrm{NiMoO}_{4}$, 说明复合材料的等效串联电阻 $\left(R_{\mathrm{S}}\right)^{[16]}$ 较小。另外, $\mathrm{NiMoO}_{4} / \mathrm{MWCNTs}-40$ 半圆半径较单组 分 $\mathrm{NiMoO}_{4}$ 更小, 表明复合材料的界面电荷转移阻抗 $\left(R_{\mathrm{ct}}\right)^{[17]}$ 更小。而在低频区, $\mathrm{NiMoO}_{4} / \mathrm{MWCNTs}-40$ 复合


图 2 (a) (e) $\mathrm{NiMoO}_{4} / \mathrm{MWCNTs}-40$ 的 EDS Mapping 图; (f) 不同 MWCNTs 含量的 $\mathrm{NiMoO}_{4} / \mathrm{MWCNTs}$ 复合材料的 XRD 图谱

Fig. 2 (a-e) EDS mapping images of $\mathrm{NiMoO}_{4} / \mathrm{MWCNTs}-40$; (f) XRD patterns of $\mathrm{NiMoO}_{4} / \mathrm{MWCNTs}$ with different amounts of MWCNTs 


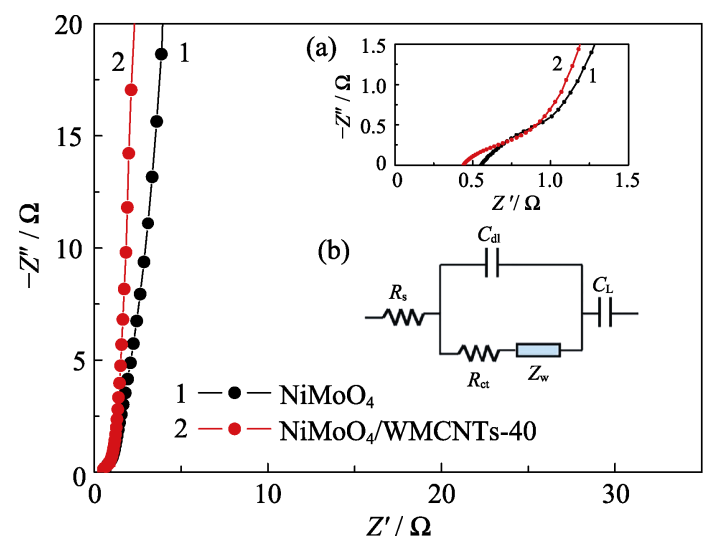

图 $3 \mathrm{NiMoO}_{4}$ 和 $\mathrm{NiMoO}_{4} / \mathrm{MWCNTs}-40$ 的交流阻抗图谱, 内插图为(a)高频放大图和(b)等效电路图

Fig. 3 EIS spectra of $\mathrm{NiMoO}_{4}$ and $\mathrm{NiMoO}_{4} / \mathrm{MWCNTs}^{-40}$ with the inset showing (a) amplified portion of the high frequency region and (b) simulated model for nyquist plots

材料的斜率明显大于 $\mathrm{NiMoO}_{4}$, 表明电荷由电解液 转移到复合材料电极表面的扩散阻抗 $\left(Z_{\mathrm{w}}\right)^{[18]}$ 更小。 根据内插图中的等效电路图进一步拟合, 得到 $\mathrm{NiMoO}_{4}$ 的 $R_{\mathrm{s}} 、 R_{\mathrm{ct}} 、 Z_{\mathrm{w}}$ 分别为 $0.54 \Omega 、 0.357 \Omega$ 和 $0.182 \mathrm{~S} \cdot \mathrm{s}^{0.5} \cdot \mathrm{cm}^{-2}$; 而 $\mathrm{NiMoO}_{4} / \mathrm{MWCNTs}-40$ 的相应阻 抗分别为 $0.48 \Omega 、 0.25 \Omega$ 和 $0.053 \mathrm{~S} \cdot \mathrm{s}^{0.5} \cdot \mathrm{cm}^{-2}$, 与前 述的定性观察相符合。阻抗分析结果说明复合材料 电极由于碳纳米管的引入而具有更为理想的电荷转 移动力学特性。

\section{3 电容测试}

进一步采用循环伏安法和恒电流充放电技术对 $\mathrm{NiMoO}_{4}$ 和 $\mathrm{NiMoO}_{4} / \mathrm{MWCNTs}$ 复合材料的电化学行 为进行表征。图 4 为加入不同量 $\operatorname{MWCNTs}(20$ 、40、 $60 \mathrm{mg}$ ) 所得复合材料的循环伏安图(a)和恒流充放电 图(b)。由图 4(a)可见, 不同 MWCNTs 含量下复合材 料的 CV 曲线都有明显的氧化还原峰, 说明其具有 噟电容特性。在相同扫速下, MWCNTs 的含量不同

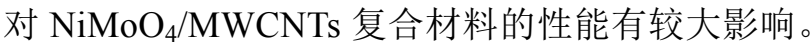
当 MWCNTs 含量为 $40 \mathrm{mg}$ 时, 得到的 $\mathrm{NiMoO}_{4} /$ MWCNTs-40 复合材料的氧化还原峰电位差最小, 峰电流最大, 说明复合材料在该组成具有最大比电 容 ${ }^{[19]}$ 。恒流充放电曲线进一步证实, 在 $2 \mathrm{~A} / \mathrm{g}$ 的恒 电流密度下, 加入 $40 \mathrm{mg}$ MWCNTs 时, 放电时间最 长, 达到 $241 \mathrm{~s}$ 。比电容和 MWCNTs 加入量的关系 曲线(图 4(b)插图)进一步表明, 随着 MWCNTs 加入 量增加, 复合材料的比电容逐渐增大, 这可解释为 材料的导电性随 MWCNTs 的增加而提高, $\mathrm{NiMoO}_{4}$ 的氧化还原反应进行的更彻底; 而当 MWCNTs 含 量超过 $40 \mathrm{mg}$ 时, $\mathrm{NiMoO}_{4} / \mathrm{MWCNTs}$ 复合材料的电 化学性能反而下降, 其原因可能是过多的 MWCNTs
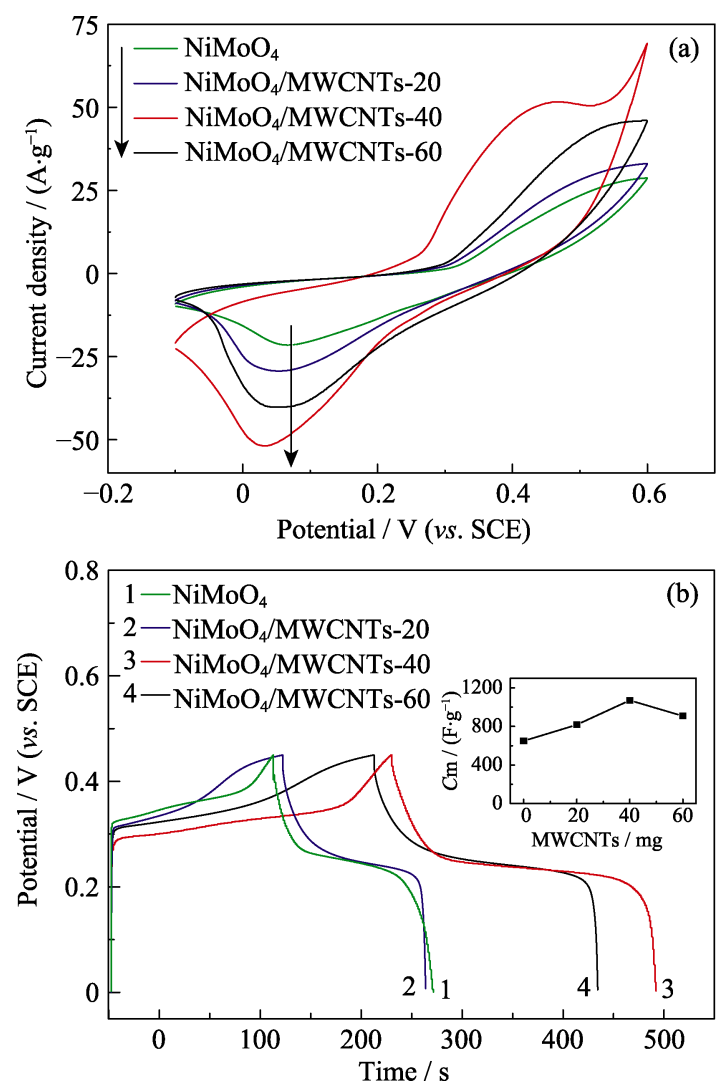

图 $4 \mathrm{NiMoO}_{4}$ 和不同 $\mathrm{MWCNTs}$ 含量的 $\mathrm{NiMoO}_{4} / \mathrm{MWCNTs}$ 复合材料的 (a)循环伏安图 (40 mV/s) 和 (b) 恒流充放电图 $(2 \mathrm{~A} / \mathrm{g})$, 插图为电容与 MWCNTs 含量关系图

Fig. 4 (a) $\mathrm{CV}$ curves $(40 \mathrm{mV} / \mathrm{s})$ and (b) GCD curves $(2 \mathrm{~A} / \mathrm{g})$ of $\mathrm{NiMoO}_{4}$ and $\mathrm{NiMoO}_{4} / \mathrm{MWCNTs}$ composites with different contents of MWCNTs with inset showing the relationship between capacitance and MWCNTs contents

阻碍了电解液向电极材料内部扩散，接触面积缩小, 使实际参与反应的活性位点减少。通过热重分析实 验，得到 $\mathrm{NiMoO}_{4}$ 在 $\mathrm{NiMoO}_{4} / \mathrm{MWCNTs}-20 、 \mathrm{NiMoO}_{4} /$ MWCNTs-40、 $\mathrm{NiMoO}_{4} / \mathrm{MWCNTs}-60$ 复合材料中的 百分比分别为 $58.22 \%, 53.55 \%, 49.23 \%$ ，呈现递减 趋势，进一步说明 MWCNTs 很好地参与了 $\mathrm{NiMoO}_{4} /$ MWCNTs 复合材料的生成。选择加入 $40 \mathrm{mg}$ MWCNTs 作为最佳条件合成的复合材料进行后续 实验。

图 5(a)为 $\mathrm{NiMoO}_{4} / \mathrm{MWCNTs}-40$ 电极材料在不 同扫描速率下的循环伏安图。由图可知，随着扫描 速度的加快, 氧化峰向正电位偏移, 还原峰向负电 位偏移，这是由于扫描速度加快，电解液中离子的 扩散速度小于电化学反应时电子的转移速度, 形成 浓差极化的结果。图 5(b)为 $\mathrm{NiMoO}_{4} / \mathrm{MWCNTs}-40$ 在不同电流密度下的恒流充放电曲线 $(G C D)$ 。由 图 5(b)可知，随着电流密度增加，电极材料的充放 电时间逐渐缩短，其原因是在高电流密度下，电极 - 电解液界面吸附大量离子, 从而导致电解液中电 

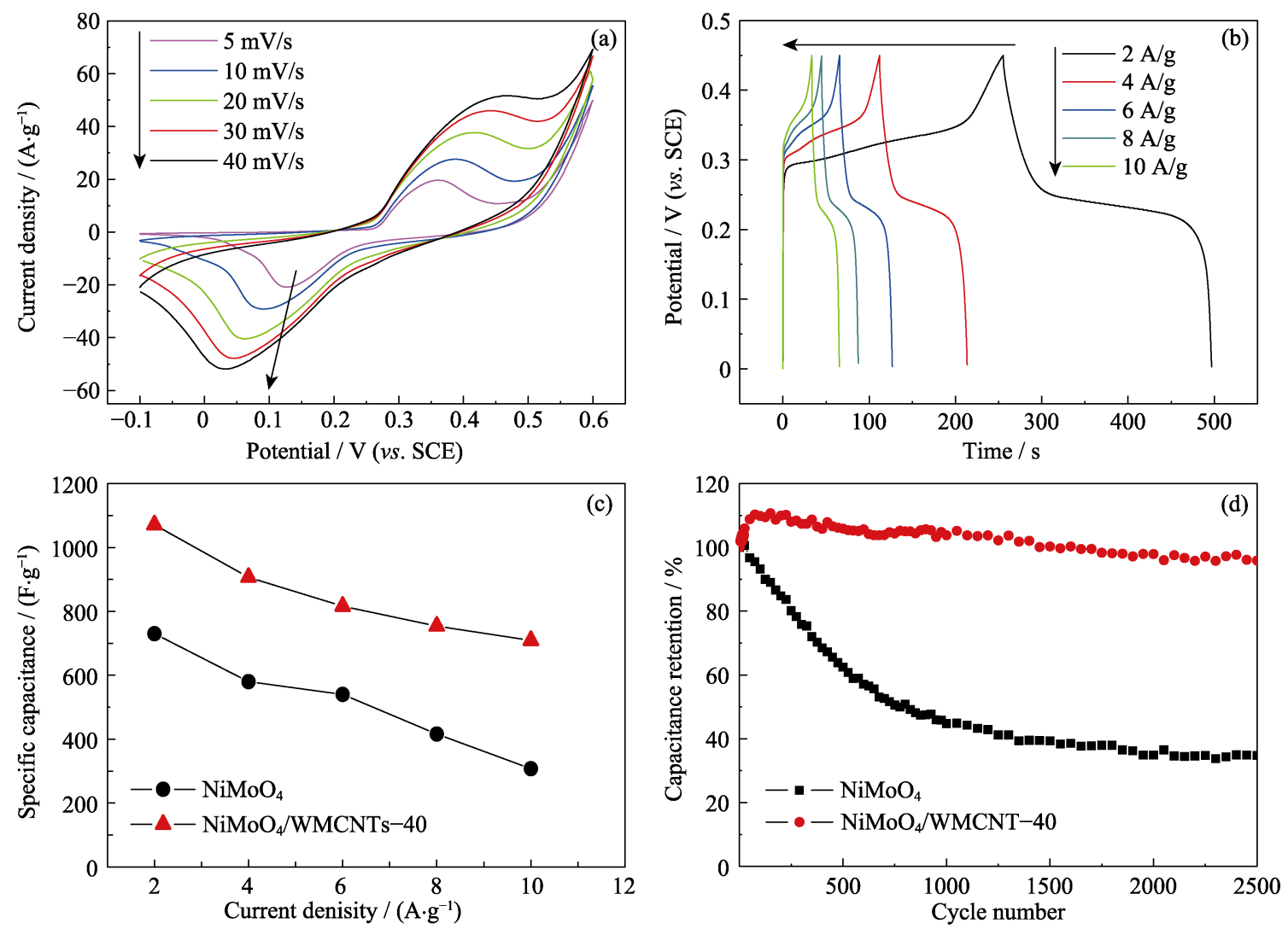

图 $5 \mathrm{NiMoO}_{4} / \mathrm{MWCNTS}-40$ 复合材料在(a)不同扫速下的循环伏安图和(b)不同电流密度下的恒电流充放电图; $\mathrm{NiMoO}_{4}$ 和 $\mathrm{NiMoO}_{4} / \mathrm{MWCNTs}-40$ 材料在(c)不同电流密度下的比容量对比图和(d)电流密度 $10 \mathrm{~A} / \mathrm{g}$ 时的循环稳定性曲线

Fig. 5 (a) CV curves and (b) GCD curves of $\mathrm{NiMoO}_{4} / \mathrm{MWCNTs}^{-40}$, (c) GCD specific capacitances of

$\mathrm{NiMoO}_{4}$ and $\mathrm{NiMoO}_{4} / \mathrm{MWCNTs} 40$ at different current densities and (d) cycling stability of $\mathrm{NiMoO}_{4}$ and NiMoO $4 / \mathrm{MWCNTs}_{4} 40$ at the current density of $10 \mathrm{~A} / \mathrm{g}$

极材料附近的离子浓度迅速下降，形成浓差极化， 难以提供足够的界面电荷以激发和维持高电流密度, 从而牺牲了部分电容, 导致比电容值下降 ${ }^{[20]}$ 。如 图 5(c)为 $\mathrm{NiMoO}_{4}$ 和 $\mathrm{NiMoO}_{4} / \mathrm{MWCNTs}-40$ 复合材料 在不同电流密度下的比电容对比图。从图 5(c)可以 更明显地看出, $\mathrm{NiMoO}_{4} / \mathrm{MWCNTs}-40$ 复合材料的性 能优于 $\mathrm{NiMoO}_{4}, \mathrm{NiMoO}_{4}$ 在 2、4、6、8、10 A/g 的 电流密度下，比容量分别是 730、580、540、416、

$307 \mathrm{~F} / \mathrm{g}$; 而 $\mathrm{NiMoO}_{4} / \mathrm{MWCNTs}-40$ 复合材料在相应 电流密度下的比电容分别是 1071、906、816、753 和 $708 \mathrm{~F} / \mathrm{g}$ 。 $\mathrm{NiMoO}_{4} / \mathrm{MWCNTs}-40$ 复合材料在不同 电流密度下的比电容均高于 $\mathrm{NiMoO}_{4}$, 说明复合材 料具有更好的电容性能。通过计算可以得到, 电流 密度从 $2 \mathrm{~A} / \mathrm{g}$ 增加到 $10 \mathrm{~A} / \mathrm{g}$ 时, $\mathrm{NiMoO}_{4} / \mathrm{MWCNTs}-$ 40 的比电容保持率为 $66.10 \%$, 远远高于 $\mathrm{NiMoO}_{4}$ 的 比电容保持率 $(42.12 \%)$, 说明 MWCNTs 的加入提高 了电极的比容量和倍率性能。

图 5(d) 是 $\mathrm{NiMoO}_{4}$ 和 $\mathrm{NiMoO}_{4} / \mathrm{MWCNTs}-40$ 电极 在电流密度为 $10 \mathrm{~A} / \mathrm{g}$ 时进行 2500 次恒流充放电的 循环稳定性曲线。实验结果表明, $\mathrm{NiMoO}_{4} / \mathrm{MWCNTs}-$
40 电极在 2500 次循环后比容量从 $708 \mathrm{~F} / \mathrm{g}$ 降到 $679 \mathrm{~F} / \mathrm{g}$ ，保持率高达 $95.85 \%$ 。而未掺杂碳纳米管的 电极经 2500 次循环后比容量从 $307 \mathrm{~F} / \mathrm{g}$ 衰减至 $107 \mathrm{~F} / \mathrm{g}$, 保持率仅有 $34.81 \%$ 。由此可知, 掺杂碳管 后材料的循环稳定性也得到了明显改善, 这主要归 功于 $\mathrm{NiMoO}_{4} / \mathrm{MWCNTs}-40$ 独特的结构: (1) MWCNTs 包覆在 $\mathrm{NiMoO}_{4}$ 外围, 形成结合力较好且均匀的纳 米结构; (2) 碳管的高比表面积和强导电性为电解 质离子的扩散和电极材料的电子传递提供了更大的 接触面积, 因而其比容量、倍率性能和循环稳定性 均有显著的提高。

\section{3 结论}

本文通过简单的水热反应法制备出低结晶度的 近球形 $\mathrm{NiMoO}_{4} / \mathrm{MWCNTS}$ 复合材料, 并将其作为超 级电容器的电极材料进行性能测试。考察了 MWCNTs 的加入对材料结构、形貌以及电化学性能的影响。 电化学充放电实验显示, 在 $2 \mathrm{~A} / \mathrm{g}$ 的电流密度下, $\mathrm{NiMoO}_{4} / \mathrm{MWCNTs}-40$ 复合材料的比电容为 $1071 \mathrm{~F} / \mathrm{g}$, 
远远高于纯 $\mathrm{NiMoO}_{4}$ 的 $730 \mathrm{~F} / \mathrm{g}$ 。循环稳定性测试表 明, 在 2500 次循环之后, $\mathrm{NiMoO}_{4} / \mathrm{MWCNTs}-40$ 电极 的电容保持率为 $95.85 \%$, 而 $\mathrm{NiMoO}_{4}$ 电极的稳定性 较低, 电容保持率仅为 $34.81 \%$ 。综上所述, 掺杂 MWCNTs 后显著提高了材料的比容量、倍率性能以 及循环稳定性。

\section{参考文献:}

[1] LIU Y, PENG X S. Recent advances of supercapacitors based on two-dimensional materials. Appl. Mater. Today, 2017, 8: 104-115.

[2] WATCHARATHARAPONG T, SUNDARAM M M, CHAKRABORTY S, et al. Effect of transition metal cations on stability enhancement for molybdate-based hybrid supercapacitor. ACS Appl. Mater. Inter., 2017, 9(21): 17977-17991.

[3] GAO S A, ZHAO J C, ZHAO Y, et al. $\mathrm{Na}_{2} \mathrm{CoSiO}_{4}$ as a novel positive electrode material for sodium-ion capacitors. Mater. Lett., 2015, 158: 300-303.

[4] WANG H W, ZHANG Y, ANG H X, et al. A high-energy lithiumion capacitor by integration of a 3D interconnected titanium carbide nanoparticle chain anode with a pyridine-derived porous nitrogen-doped carbon cathode. Adv. Funct. Mater., 2016, 26(18): 3082-3093.

[5] GONZALEZ A, GOIKOLEA E, BARRENA J A, et al. Review on supercapacitors: technologies and materials. Renew. Sust. Energ. Rev., 2016, 58: 1189-1206.

[6] CAI D P, WANG D D, LIU B, et al. Comparison of the electrochemical performance of $\mathrm{NiMoO}_{4}$ nanorods and hierarchical nanospheres for supercapacitor applications. ACS Appl. Mater. Inter., 2013, 5(24): 12905-12910.

[7] YUAN W, HAN G Y, XIAO Y M, et al. Flexible electrochemical capacitors based on polypyrrole/carbon fibers via chemical polymerization of pyrrole vapor. Appl. Surf. Sci., 2016, 337: 274-282.

[8] ZHANG J, TERRONES M, RAC PARK C, et al. Carbon science in 2016: status, challenges and perspectives. Carbon, 2016, 98: 708-732.

[9] ZHENG X, GONG C L, LIU H, et al. Preparation of phosphomolybdic acid coated carbon nanotubes and its supercapacitive properties. J. Inorg. Mater., 2017, 32(2): 127-134.
[10] LIU T, CHAI H, JIA D, et al. Rapid mircowave-assisted synthesis of mesoporous $\mathrm{NiMoO}_{4}$ nanorod/reduced graphene oxide composites for high-performance supercapacitors. Electrochim. Acta, 2015, 180: $998-1006$.

[11] MEFFORD J T, HARDIN W G, DAI S, et al. Stevenson anion charge storage through oxygen intercalation in $\mathrm{LaMnO}_{3}$ perovskite pseudocapacitor electrodes. Nat. Mater., 2014, 13(7): 726-732.

[12] LOTA G, FIC K, FRACKOWIAK E. Carbon nanotubes and thek composites in electrochemical applications. Energ. Environ. Sci., 2011, 4(5): 1592-1605.

[13] ZHOU H, ZHANG L S, ZHANG D Y, et al. A universal synthetic route to carbon nanotube/transition metal oxide nano-composites for lithium ion batteries and electrochemical capacitors. Sci. RepUK., 2016, 6: 37752-1-11.

[14] FARES M M, ABU AL-RUB F A, MASSADEH K H. Lightweight MWCNTs-g-PAN carbon fiber precursors. Sensitive high absorptivity and novel wide-bandgap conjugated polymers. Ind. Eng. Chem. Res., 2015, 54(37): 9064-9071.

[15] OKPALUGO T I T, PAPAKONSTANTINOU P, MURPHY H, et al. High resolution XPS characterization of chemical functionalised MWCNTs and SWCNTs. Carbon, 2005, 43(1): 153-161.

[16] ZHOU Y J, MAO Z M, WANG W, et al. In-situ fabrication of graphene oxide hybrid Ni-based metal-organic framework (NiMOFs@GO) with ultrahigh capacitance as electrochemical pseudocapacitor materials. ACS Appl. Mater. Inter., 2016, 8(42): 28904-28916.

[17] BENOIT C, DEMETER D, BELANGER D, et al. A redox-active binder for electrochemical capacitor electrode. Angew. Chem. Int. Ed., 2016, 128(17): 1-5.

[18] WU C, CAI J J, ZHANG Q B, et al. Hierarchical mesoporous zinc-nickel-cobalt ternary oxide nanowire arrays on nickel foam as high-performance electrodes for supercapacitors. ACS Appl. Mater. Inter., 2015, 7(48): 26512-26521.

[19] ZHU P, CAI T, HAN G Y, et al. Preparation and electrochemical performance of $\mathrm{PPy} / \mathrm{GO}-\mathrm{RuO}_{2}$ film electrode for micro-supercapacitor. J. Inorg. Mater., 2015, 30(5): 505-510.

[20] ZHOU Y J, MAO Z M, WANG W, et al. In-situ fabrication of graphene oxide hybrid Ni-based metal-organic framework (NiMOFs@GO) with ultrahigh capacitance as electrochemical pseudocapacior materials. ACS Appl. Mater. Inter., 2016, 8(42): 28904 28916. 